\author{
${ }^{1}$ Icahn School of Medicine at Mount Sinai, New York, NY, \\ USA \\ ${ }^{2}$ Centers for Disease Control and Prevention/National Insti- \\ tute for Occupational Safety and Health, Atlanta, GA, USA
}

\section{8a THE CONTRIBUTION OF OCCUPATIONAL MEDICINE TO EMERGENCY PREPAREDNESS: NEW YORK CITY AND 9/ 11}

Philip J Landrigan. Icahn School of Medicine at Mount Sinai, New York, NY, USA

\subsection{6/oemed-2018-ICOHabstracts.319}

Background Occupational exposures to hazardous materials and to high levels of psychological stress are predictable consequences of civilian disasters and result commonly in physical and mental health problems among first responders. Emergency plans must anticipate these occupational health consequences of disaster and be prepared to address them.

Goal To assess the contribution of occupational medicine to emergency response after the attacks on the World Trade Centre of September 11, 2001.

Method Historical review

Findings New York had a network of Centres of Excellence in Occupational Health in place prior to September 11, 2001. These Centres were established in 1987, supported financially by the State of New York, and focused on the prevention, diagnosis, and treatment of injuries and illnesses among workers. They were staffed by trained personnel and provided a wide range of services including occupational medicine, occupational health nursing, industrial hygiene, ergonomics, and social work services.

The New York Centres of Excellence in Occupational Health were able to provide medical care to first responders immediately after September 11, 2001. Within a few weeks they had evaluated several hundred responders - firefighters, police, paramedics, and volunteers. Persistent cough, termed 'World Trade Centre cough', asthma, anxiety, depression, and post-traumatic stress disorder (PTSD) were the symptoms most commonly seen in the initial post-attack period. These symptoms were most severe in the most heavily exposed responders and were more frequent among 9/11 first responders than among unexposed workers in the same trades.

Documentation of these health effects and their persistence was critical in demonstrating that the attacks on the World Trade Centre and their aftermath had produced physical and mental health effects among first responders; in establishing the need for long-term medical and epidemiologic follow-up of the first responder population; and in ensuring that this follow-up included comprehensive diagnosis and treatment of both physical and mental health conditions. Follow-up of the 9/11 responders continues to the present, is supported by the US government through the National Institute for Occupational Safety and Health (NIOSH), and has documented multiple exposure-related health problems in this population including restrictive lung disease, chronic sinusitis, gastroesophageal reflux disease (GERD), PTSD, depression, and cancer.

Conclusion Pre-positioned resources in occupational medicine are an essential component of disaster response. Emergency preparedness planning must include long-term investments in occupational and environmental health services.
$1608 b$

INDICATIONS FOR LIFETIME HEALTHCARE OF AFFECTED POPULATIONS FROM THE WORLD TRADE CENTRE AND OTHER MAJOR INTERNATIONAL DISASTERS

Roberto G Lucchini. Icahn School of Medicine at Mount Sinai, New York, NY, USA

10.1136/oemed-2018-ICOHabstracts.320

Background The disasters at Seveso, Three Mile Island, Bhopal, Chernobyl, the World Trade Centre (WTC) and Fukushima had historic health and economic sequelae for large populations of workers, responders and community members.

Methods Comparative data from these events were collected to derive indications for future preparedness. Information from the primary sources and a literature review addressed:

i. exposure assessment;

ii. exposed populations;

iii. health surveillance;

iv. follow-up and research outputs;

v. observed physical and mental health effects;

vi. treatment and benefits; and

vii. outreach activities.

Results Exposure assessment was conducted in Seveso, Chernobyl and Fukushima, although none benefited from a timely or systematic strategy, yielding immediate and sequential measurements after the disaster. Identification of exposed subjects was overall underestimated. Health surveillance, treatment and follow-up research were implemented in Seveso, Chernobyl, Fukushima, and at the WTC, mostly focusing on the workers and responders, and to a lesser extent on residents. Exposurerelated physical and mental health consequences were identified, indicating the need for a long-term health care of the affected populations. Fukushima has generated the largest scientific output so far, followed by the WTCHP and Chernobyl. Benefits programs and active outreach figured prominently in only the WTC Health Program. The analysis of these programs yielded the following lessons:

- Know who was there;

- Have public health input to the disaster response;

- Collect health and needs data rapidly;

- Take care of the affected;

- Emergency preparedness;

- Data driven, needs assessment, advocacy.

Conclusion Given the long-lasting health consequences of natural and man-made disasters, health surveillance and treatment programs are critical for management of health conditions, and emergency preparedness plans are needed to prevent or minimise the impact of future threats.

\section{C RESPIRATORY DISEASE RESEARCH IN THE WTC HEALTH PROGRAM}

Rafael E de la Hoz. Icahn School of Medicine at Mount Sinai, New York, NY, USA

10.1136/oemed-2018-ICOHabstracts.321

The terrorist attack on 9/11/2001, and subsequent rescue, recovery, and service restoration of the World Trade Centre disaster site in New York City, created an unprecedented and unique occupational and environmental exposure that affected a large and diverse group of rescue workers and volunteers. 